\author{
YVES GAMBIER
}

Université de Turku, Finlande

\title{
TRADUCTION ET ANALYSES DE DISCOURS: TYPOLOGIE CROISÉE
}

\begin{abstract}
Gambier Yves, Traduction et analyses de discours: typologie croisée [Translation and analysis of discourse: crossed typology]. Studia Romanica Posnaniensia, Adam Mickiewicz University Press, Poznań, vol. XXV/XXVI: 2000, pp. 97-108. ISBN 83-232-0965-0. ISSN 0137-2475.

The article is focussed on tracing all the relations which have existed between science on language and the study of translation. The author makes an attempt at showing in what way the reflection on the theory of discourse may influence translation and, in consequence, on the way translators are instructed.
\end{abstract}

Les analyses de discours (AD) peuvent être des outils utiles à l'apprenant-traducteur, et peut-être même des outils conceptuels pertinents pour la théorisation de la traduction. Mais je m'en tiendrai à la première visée. Je n'aurai pas l'outrecuidance de détailler l'histoire des rapports entre sciences du langage et science de la traduction. Cependant une certaine mise en perspective, avec quelques références à titre d'exemples, devrait aider à mieux situer les enjeux théoriques et pédagogiques ainsi qu'à mieux souligner les sources de certains malentendus dans nos efforts réciproques d'interdisciplinarité.

\section{LINGUISTIQUE ET TRADUCTION}

Les rapports entre linguistique et traduction ont longtemps été marqués par l'ignorance mutuelle, sinon l'exclusion hautaine. Jusqu'à la fin des années 60 , la linguistique était assimilée au structuralisme et à la théorie générative. Certains auteurs ont tenté d'asseoir leurs réflexions générales, méthodologiques en traduction sur cette linguistique de la «langue» (Catford 1965, Mounin 1976, Koptjevskaja-Mamm 1989, 
etc.). Prédominait alors une conception de la traduction comme transfert, comparaison de structures, indépendante de toute dimension pragmatique, sociolinguistique, discursive. Au même moment, cette vision un peu mécaniste des langues et des traductions était renforcée par l'utopie de la traduction automatique. Cette étape formelle a été bousculée par une étape qu'on pourrait appeler ethno-sémantique: il y a eu d'une part retour du refoulé en linguistique c'est-à-dire de la problématique du sens et d'autre part appréhension deș aspects culturels de la signification grâce à certains travaux anthropologiques (Boas, Malinowski, Sapir, Lévi-Strauss, etc.). Nida illustre assez bien cet itinéraire: parti de la grammaire transformationnelle (1964), il en vient aux analyses componentielles, sémiques, pour peu à peu intégrer les dimensions sociales et culturelles (avec Taber 1969). La linguistique contrastive elle-même a connu des évolutions: tantôt héritière d'une tradition pure et dure, tantôt allant au-delà de la proposition (Vinay-Darbelnet 1958; Guillemin-Flescher 1981), se mettant parfois dans un cadre théorique précis - cf. par exemple la systématique comparée de Garnier (1985) appliquant la psychomécanique du langage due à G. Guillaume.

Le problème du sens a certainement bousculé les conceptions en traduction. Ce n'est pas le lieu de rappeler les différents modèles qui ont été discutés en traductologie: de la sémantique structurale, à laquelle on peut rattacher les notions de «shift» ou «transposition», à la sémantique de Fillmore (scenes/frames) qui fait dépendre le sens d'un mot du contexte et du type de communication, en passant par la sémantique des prototypes, l'approche socio-sémiotique du sens... il a fallu s'interroger sur les composantes, le processus, les conditions pour «faire sens» quand on écrit, quand on parle ou interagit. Divers modèles ont été proposés. Quoi qu'il en soit, les traductologues ont été confrontés à des concepts comme communication, situation, contexte, message, intentionalité, réception, etc. - ébranlant les positions naguère figées sur l'équivalence entre textes de départ et d'arrivée, la fidélité...

Linguistique et traduction: quel est désormais le sens de cet ordre des mots? Qu'implique la conjonction «et»? La coordination? La subordination? La complémentarité? L'inclusion? Deux remarques s'imposent ici:

- Le linguiste propose des analyses, rend compte de fonctionnements langagiers tandis que le traducteur a une démarche finalisée: il prend une suite de décisions pour arriver à un produit qui satisfasse les objectifs assignés par un commanditaire. Quant au traductologue, il s'efforce d'expliquer les spécificités et les contraintes de la communication interlinguistique.

- La linguistique s'est élargie pour devenir sciences du langage. Le passage d'un générique singulier au pluriel n'est pas un accident. Il ne s'agit pas d'un domaine monolithique ni statique: les écoles et tendances y foisonnent dorénavant et surtout les objets d'analyse, les méthodologies, les présupposés... y sont divers. On ne peut que s'étonner dès lors que certains en traductologie aient jeté le bébé avec l'eau du bain, contestant la légitimité sinon la nécessité de la composante linguistique dans l'approche de la traduction, en se bloquant sur un état daté de la recherche linguistique 
(réduite au formalisme des années 60). Par ailleurs, des linguistes continuent d'amalgamer la traduction à la version et au thème, familiers de l'apprentissage des langues, ou encore à confondre traduction avec linguistique contrastive. Rares sont les chercheurs qui comme Culioli, Bastuji (1974), admettent les limites de leurs efforts pour couvrir la traduction. Ces malentendus cachent parfois des questions de champ, sinon de pouvoir. Paradoxe complémentaire: les rejets a priori n'excluent pas les plaidoyers pour l'interdisciplinarité!

L'élargissement des préoccupations et des moyens de la linguistique comme l'élargissement des problèmes ou variables à traiter pour mieux comprendre la traduction, comme processus et comme produit, ont convergé peu à peu. Les réflexions sémantiques des traducteurs ont ainsi amené à croiser les analyses pragmatiques, les propositions de la linguistique textuelle, de la rhétorique, les hypothèses et les moyens des AD, de la linguistique cognitive, etc. Depuis la fin des années 70 , le texte comme unité de traduction, les types de texte à traduire, l'importance du contexte et du cotexte (ou intertextualité) pour saisir puis pour rendre un message (littéraire, technique, juridique, commercial, etc.) ont renouvelé les approches traductologiques: on peut citer ici aussi bien l'«école de Leipzig» (avec Neubert, Kade, Jäger) que l'«école de la manipulation» (avec Lefevere, Lambert) ou des chercheurs isolés (Holmes; Toury par ex.) qui ont développé des approches systématiques, descriptives... On ne compte plus les publications abordant la traduction comme une communication complexe, offrant différents niveaux d'analyse et référant à des travaux élaborés en sciences du langage. Les apports de la théorie des actes de langage, du concept de texte comme suite non-linéaire d'éléments, du principe de coopération, et des maximes qui en découlent, du principe de pertinence... ont fécondé les analyses de (par exemple) Blum-Kulka (1981), Tirkkonen-Condit (1986), Gutt (1991).

La linguistique textuelle, soucieuse de l'organisation d'ensemble d'un texte, de sa progression thématique, de sa texture proprement dite (cohérence; cohésion) a aussi alimenté bien des efforts d'investigation - par exemple Neubert (1985), Hatim et Mason (1990; 1997), Nord (1991), etc.

Le tournant dit culturel en traductologie (années 80) n'a pas rendu obsolètes toutes ces réflexions théoriques, méthodologiques, didactiques. Chomskyenne, fonctionnelle (avec le cercle de Prague), systémique (avec Halliday), pragmatique, textuelle, cognitive (cf. Tabakowska, 1993), «la» linguistique ne cesse de renouveler nos perceptions de la traduction. Il est dommage que traducteurs et traductologues ne fassent guère de remarques sur l'hétérogénéité structurée des langues, sur les variations textuelles, sur les enjeux culturels et identitaires de la communication verbale, alors même qu'ils sont confrontés aux tensions interlinguistiques, interculturels et que leurs observations pourraient souvent enrichir, parfois contrecarrer, les hypothèses, les descriptions, les explications - élaborées à partir de corpus majoritairement monolingues. 


\section{PROPOSITIONS TYPOLOGIQUES}

Depuis Aristote... jusqu'aux étiquettes socio-commerciales d'aujourd'hui, chercheurs, auteurs de textes (y compris les traducteurs), usagers ont eu besoin de catégoriser textes et discours. Les études littéraires, rhétoriques, en folkloristique, en bibliothéconomie, et plus récemment en langues de spécialité ont eu recours à diverses typologies pour organiser la masse des productions écrites et orales. On ne peut guère présentement même résumer les débats sur les critères, la pertinence des regroupements proposés ni les polémiques sur les rapports éventuels entre types, genres, registres (cf. Trosborg, 1997). On admettra ici qu'un manuel d'entretien, un mode d'emploi, une lettre d'affaires, un roman policier, un article de presse, une pièce radiophonique, une page d'Internet... créent des attentes: ce sont des «genres» définis à priori, des pré-textes en quelque sorte qui orientent notre réception, tandis que les «types» (savant, polémique, vulgarisateur, didactique, informatif, argumentatif, etc.) sont dégagés a posteriori, suite à notre lecture, à partir de certains signes linguistiques. Genres et types, déterminés par des conventions, des traditions, des normes déterminent à leur tour des contraintes de production et d'interprétation. Un juriste qui plaiderait en vers se ferait sanctionner par l'Ordre des avocats; un scientifique qui voudrait publier dans une revue un exposé rédigé comme une recette de cuisine se heurterait au Comité de lecture.

Quelques typologies ont marqué certains travaux en linguistique textuelle (cf. Rolf, 1993). On citera:

- A. Bain (1890) qui a défini cinq modes de discours (descriptif, narratif, expositif, argumentatif et poétique);

- R. Jakobson (1960) dont les six fonctions du langage (expressive, conative, phatique, poétique, référentielle, métalinguistique) ont donné lieu à des essais de différenciation typologique;

- J. Kinneary (1971: A Theory of Discourse: The Aims of Discourse, 1980: A Theory of Discourse), amené à discerner les discours expressifs, persuasifs, referentiels (ou expositifs) et littéraires;

- R. Longacre (1976: An Anatomy of Speech Notions) qui a distingué entre six caractéristiques essentielles (narrative, procédurale, expositive, descriptive, dramatique et incitative);

- E. Werlich (1976: A Text Grammar of English), célèbre pour ses cinq distinctions (description, narration, argumentation, exposition, instruction);

- L. Lundqvist (1983: L'analyse textuelle) qui a répertorié sept «formes de représentation» pour classer les discours (expressive, informative, scénique, narrative, descriptive, argumentative, directive);

- J.M. Adam (1985a: Le texte narratif; 1985b: Quels types de textes?, Le Français dans le monde 192 (p. 39-43); 1992: Les textes: types et prototypes) développant huit types (descriptif, narratif, explicatif, argumentatif, injonctif (ou instructif), prédictif, conversationnel et rhétorique). 
Quelques remarques générales s'imposent:

- derrière les mêmes appellations, se cachent parfois des notions partiellement différentes;

- les critères de classification utilisés par les auteurs sont hétérogènes et même inégalement exploités chez le mểme auteur;

- un même genre (par ex. une fable) peut mêler divers types, même si l'un d'entre peut dominer. Cette pluralité de types pour une entité (écrite ou orale) donnée font que les catégories suggérées sont flexibles. N'empêche, un auteur ou un lecteur saura se placer dans une lignée d'usages codifiés, pour se conformer à ou transgresser ou plagier un «genre» ou un «type». On sait aussi qu'historiquement les «mauvais genres» peuvent acquérir droit de cité, reconnaissance institutionnelle (ex. BD, littérature dite pour enfants, science-fiction, etc.). Ce qui compte dans notre perspective, c'est que l'apprenant-traducteur sache convenir des genres et des types du document qu'il a à traduire, qu'en conséquence il sache appliquer certains règles d'interprétation et de production textuelle et qu'il sache percevoir les différences éventuelles avec ses propres habitudes. Un éditorial (genre) est argumentatif en français mais plutôt informatif en finnois (c.-à̀-d. ne donnant pas une prise de position de la rédaction).

En traduction aussi, on a tenté de classifier les textes habituellement à traduire soit pour des raisons théoriques, soit à des fins d'enseignement. Divers paramètres ont été également utilisés (cf. Hurtado Alibir, 1996):

- le domaine (d'où les différenciations thématiques entre traductions biblique, scientifique, commerciale, médicale, juridique, littéraire, etc.). De tels macro-domaines appellent des sous-domaines (par ex. traduction littéraire $>$ traduction poétique, dramatique, de livres d'enfants, de littérature de gare, etc.);

- les fonctions ou types textuels: en général, on a alors des tripartitions entre textes expressifs, informatifs et opératifs (par ex. Reiss, 1976; Newmark, 1981) ou des distictions binaires (traduction littéraire vs traduction pragmatique / Delisle, 1980);

- les façons de traduire, d'où les oppositions entre traduction littérale et traduction libre; entre traduction du sens et traduction de la forme... et autres dichotomies sans retombées théoriques ou didactiques - comme encore par ex. l'opposition entre traduction sémantique, sourcière (centrée sur l'auteur, le texte de départ) et traduction communicative, cibliste (pliée aux normes d'arrivée).

- le degré de traduisibilité: par ex. J. House (1981) qui a separé traduction explicite (overt translation) et traduction cachée (covert translation) jouissant de la condition d'original dans la culture d'arrivée.

Comme les typologies en linguistique textuelle, les typologies en traduction tendent à figer les catégories, à ne considérer que certaines variables au détriment d'autres, à mêler des critères de différente nature (linguistique, thématique, fonctionnelle, etc.); surtout, elles hésitent entre texte de départ et texte d'arrivée, l'accent étant placé tantôt sur l'un, tantôt sur l'autre. 


\section{APPROCHES DISCURSIVES DE LA TRADUCTION}

Avec ce qui précède, on peut maintenant préciser les nécessités de l'approche discursive et cela pour quatre raisons au moins, qui peuvent paraître triviales à des linguistes mais qui ont exigé efforts chez les traducteurs/traductologues.

a. Divers modèles du processus de traduction ont été proposés. Par exemple celui en trois étapes (appréhension du texte de départ/déverbalisation/réexpression en texte d'arrivée), formulé encore dans le cadre des recherces en traduction automatique sous la forme: analyse/transfert (ou recherche d'équivalences)/génération (output). On admettra que ces modèles ne considèrent que la traduction au sens étroit du terme, négligeant par exemple la réception, la planification du travail, la lecture et la recherche documentaire/terminologique, les révisions successives, la relecture finale, la mise en page, la livraison, la facturation, etc. - phases qui soulignent que le traducteur ne travaille pas dans une tour d'ivoire mais pour répondre à une commande ciblée, embrayée sur des besoins à satisfaire. L'opposition traductologie à orientation linguistique - traductologie à orientation fonctionnelle (Snell-Hornby, 1988:14) est désormais arbitraire et non pertinente: non seulement parce qu'il y a d'autres approches (féministe, déconstructionniste, descriptive, post-colonialiste, etc.) mais parce que personne ne peut plus s'en tenir à un concept d'équivalence ambigu, amorphe, polysémique, prétendument basé sur les seuls traits structuraux du texte de départ. Toute texte ou discours prend forme et sens

- en situation, elle-même inscrite dans une culture donnée;

- par rapport à d'autres textes ou discours, immédiats ou appartenent au patrimoine de la communauté;

- en fonction des attentes du client (qui commande la traduction) et des futurs récepteurs de cette traduction.

Dans la dynamique complexe qui vient d'être rappelée, la phase de la compréhension est une des phases importantes. La compréhension du texte de départ est liée à la compréhension des raisons du commanditaire, à celle des normes et conventions établies qui font que le texte de départ est composé comme il est: au niveau explicite de sa macro-structure, au niveau implicite (allusions, présupposés, sous-entendus, euphémismes, etc.). Les textes de départ et d'arrivée ne sont pas indépendants de leurs conditions d'énonciation.

b. Quand il traduit, le traducteur est sensible à la complexité des rapports entre les variétés linguistiques et les groupes sociaux, au rôle du prestige et de la stigmatisation des niveaux de langue, aux mécanismes de diffusion des innovations (linguistiques, littéraires...), aux interférences produites par le contact des langues. Il suit délibérément ou pas, consciemment ou pas, tout un ensemble de «normes» pour que son travail soit reconnu, accepté. Toury (1995) a distingué deux sortes de «normes»: celles «préliminaires», touchant les critères et les manières de sélectionner les textes à traduire et celles «opérationnelles», englobant les décisions à faire pendant qu'on traduit, décisions marquées par la compréhension de la situation, des raisons de tra- 
duire, par la compréhension du texte à traduire, par la compréhension des conditions de réception à venir, etc. Les obligations éditoriales par exemple et les déterminations proprement textuelles influent sur les manières de rendre le texte d'arrivée.

Les «normes» en question, qui n'ont rien à voir avec des normes prescriptives (trop longtemps dominantes dans les discours sur la traduction) peuvent être en compétition, conflictuelles ou pas; elles peuvent être canoniques, codifiées, institutionnalisées même ou à peine formulées, reconstruites historiquement après coup. Elles disent que le comportement traductionnel est réglé, social, que la traduction fonctionne en partie par rapport à des attentes, que les stratégies du traducteur sont largement prévisibles (cf. Chesterman, 1997: ch.3).

C'est à la fois le travail de celui/celle qui convertit linguistiquement et la traduction comme produit reçu en un lieu et temps donnés qui sont interactifs. D'où les éclairages possibles de la psycho- et de la sociolinguistique (incluant les $\mathrm{AD}$ ).

c. Un ensemble de recherches actuelles, centrées sur des corpus de textes traduits, confirme que la traduction induit une langue hybride, qui n'est pas la résultante d'interférences, de calques... Ces traits sont spécifiques parce qu'ils ne sont pas ordinairement présents en quantité dans les textes rédigés directement en langue d'arrivée. C'est dire qu'un professionnel écrivant puis traduisant dans sa langue recourra à des tournures particulières différentes, préférera certaines constructions à d'autres, utilisera plus fréquemment des marques à la place d'autres, etc. De telles occurrences autorisent à penser que texte de médiation, la traduction «se sent» nécessairement, sans qu'il s'agisse là d'un jugement de valeur équivalent à «mauvais». L'analyse empirique de divers corpus, notamment à Manchester, depuis 1993 (avec M. Baker, S. Laviosa et autres), confirme que ces traits traductionnels apparaissent indépendamment des langues de départ. Il y a là un riche paradigme avec données informatisées nombreuses, méthodes rigoreuses empruntant à la linguistique - tissant un nouveau lien entre traductologie et sciences de langage.

D'autres types de recherche renforce ce lien: on citera les traitements de textes parallèles en langues de départ et d'arrivée, pour réaliser des travaux terminologiques bilingues, des systèmes de vérification de traduction, des logiciels de mémoire de traduction, etc. Le bitexte ou banque de données bitextuelles (textes-sources alignés de façon juxtalinéaire ou en parallèle avec leur(s) traduction(s)) devrait aussi faciliter la mise en oeuvre de projets de traduction assistée par ordinateur basés sur des productions authentiques et non plus exclusivement sur des calculs formels. Il pourrait inspirer également des analystes de discours.

d. Pour terminer sur la validité de l'approche discursive de la traduction, on citera la problématique de la qualité - objet de nombreuses interventions de praticiens et de décideurs, ces trois-quatre dernières années. En effet la «qualité» implique de tenir compte des acteurs qui interviennent dans la traduction (le traducteur n'étant que l'un d'eux); elle implique également de tenir compte des divers paramètres et variables qui affectent le processus et le produit de traduction (cf. a et b, ci-dessus). 
Ainsi, dans les modèles décomposant le travail (a), dans les «normes» qui régissent ce travail (b), dans les recherches sur la «langue traduite» (c), enfin dans les réflexions sur la qualité (d), on perçoit combien désormais la traduction peut être étudiée comme discours c'est-à-dire énoncé et énonciation, formation discursive (cf. Foucault, 1971; Pêcheux, 1975), intrication d'un mode de dire et d'un lieu socioculturel déterminés.

\section{IMPLICATION PÉDAGOGIQUE}

Dans ce qui suit, nous n'aurons en tête que la compréhension du texte de départ, essentielle pour rendre de manière adéquate la traduction. Divers hypothèses découlent de cette assertion. Comprendre en vue de traduire n'a pas les mềmes exigences que comprendre pour extraire une information par exemple. En outre, la compréhension n'est pas une phase autonome dans le processus traductionnel (malgré certains modèles qui découpent ce processus en étapes bien tranchées): la reformulation en langue d'arrivée pour un public donné, exige parfois de réinterpréter son texte ou un paragraphe. Linguistes, logiciens, psycholinguistes, sémioticiens, neurolinguistes, cogniticiens se sont penchés, à des titres divers et pour des visées variables, sur la lecture (compréhension, calcul de sens, interprétation). En traductologie et en pédagogie de la traduction, la compréhension n'a pas encore donné lieu à beaucoup de travaux empiriques, expérimentaux (cf. Dancette, 1995). Le plus souvent, on a des affirmations non démontrées, des répétitions de présupposés (du genre: il y a «un» sens, stable, caché dans le texte, immuable), ou encore référence à une philosophie du sens (herméneutique de Gadamer par ex., ou citations de Heidegger): la traduction consisterait à rendre «tout le sens». Avec un tel postulat, la formation des traducteurs ne marque aucune avancée. Très souvent aussi, la compréhension se réduit à la consignation de fautes de sens!

Un traducteur cherche à comprendre son texte en langue de départ en fonction des finalités de la traduction (extraire une information; mettre en scène le texte; parvenir à un accord commercial; etc.) qu'on lui a demandé de produire et qu'il doit rendre selon certains critères d'acceptabilité. Il tentera donc tuujours de circonscrire:

- la situation de communication qui détermine par exemple l'usage des interjections, des allusions, des sous-entendus, des actes de discours indirects;

- les références, exprimées par des mots ou termes mais aussi par des noms propres, des expressions culturelles;

- les marques de temps et de localisation, pour saisir les éléments déictiques, pour éviter les archaïsmes ou les anachronismes;

- les récepteurs du texte de départ (avec leurs connaissances présupposées, leurs attentes, leurs habitudes, leurs clichés, leur registre de langue, etc.)

- les valeurs attribuées au genre du texte en question; à son support de diffusion. 
Par exemple, un document de type «instructif» peut être soit subjectif (dépendant de l'autorité de son émetteur - discours politique, sermon, commentaires...), soit objectif, donnant des conseils pratiques (guide, manuel, notice technique) ou des ordres (contrats, règles d'un jeu, traités...).

Tous ces déterminants affectent le degré de compréhension du traducteur et ses choix stratégiques de traduction - que ce soit pour un manuel d'utilisation d'un logiciel, une lettre commerciale... ou un roman, un certificat de naissance...

\begin{tabular}{|c|c|c|c|c|}
\hline \multirow{3}{*}{ Types d'AD } & \multicolumn{4}{|c|}{ GENRES DE TEXTE À TRADUIRE } \\
\hline & \multirow{2}{*}{$\begin{array}{l}\text { TEXTES ÉCRITS } \\
\text { CONTINUS } \\
\text { (traduction écrite, } \\
\text { traduction à vue...) }\end{array}$} & \multirow{2}{*}{$\begin{array}{l}\text { DISCOURS } \\
\text { ORAUX ou } \\
\text { ORALISÉS } \\
\text { (interprétation) }\end{array}$} & \multicolumn{2}{|c|}{$\begin{array}{l}\text { TEXTES MULTI- } \\
\text { SÉMIOTIQUES }\end{array}$} \\
\hline & & & $\begin{array}{l}\text { SCRIPTO- } \\
\text { VISUELS }\end{array}$ & $\begin{array}{l}\text { SCRIPTO- } \\
\text { SONORES }\end{array}$ \\
\hline RHÉTORIQUE & ex. textes juridiques & & $\begin{array}{l}\text { publicités } \\
\text { BD }\end{array}$ & \\
\hline $\begin{array}{c}\text { TH. DE L'ARGUMEN- } \\
\text { TATION }\end{array}$ & discours politiques & $\begin{array}{c}\text { exposés/ } \\
\text { conférences } \\
\text { scientifiques }\end{array}$ & & \\
\hline $\begin{array}{c}\text { MODĚLE DE TRAITEMENT } \\
\text { de l'info (cf. Kinsch, } \\
\text { van Dijk, 1975, 1983) }\end{array}$ & $\begin{array}{c}\text { articles de presse, } \\
\text { éditoriaux }\end{array}$ & & & \\
\hline PRAGMATIQUE & $\begin{array}{c}\text { brochures, } \\
\text { modes d'emploi }\end{array}$ & $\begin{array}{l}\text { discours } \\
\text { politiques }\end{array}$ & $\begin{array}{l}\text { dialogues } \\
\text { audio- } \\
\text { visuels } \\
\end{array}$ & \\
\hline LEXICOMÉTRIE & textes spécialisés & & & \\
\hline $\begin{array}{l}\text { LINGUISTIQUE } \\
\text { TEXTUELLE }\end{array}$ & $\begin{array}{l}\text { textes littéraires, } \\
\text { publicitaires, } \\
\text { scientifiques }\end{array}$ & & & chansons \\
\hline SĚMIOTIQUE LITTÉRAIRE & textes religieux & & $\begin{array}{r}\text { livres } \\
\text { d'enfant }\end{array}$ & $\begin{array}{l}\text { livrets } \\
\text { d'opéra }\end{array}$ \\
\hline $\begin{array}{l}\text { SÉMIOTIQUE du visuel... } \\
\text { (non-verbal) }\end{array}$ & & & $\begin{array}{l}\text { brochures; } \\
\text { Bd; films, } \\
\text { livres ill. }\end{array}$ & CD-Rom \\
\hline $\begin{array}{l}\text { LINGUISTIQUE de } \\
\text { L'ÉNONCIATION }\end{array}$ & réclames & $\begin{array}{c}\text { débats, } \\
\text { tables rondes }\end{array}$ & $\begin{array}{l}\text { réclames, } \\
\text { progr. TV }\end{array}$ & chansons \\
\hline PRAXÉMATIQUE & $\begin{array}{c}\text { publicités } \\
\text { textes culturels } \\
\text { théoriques (essais) }\end{array}$ & & $\begin{array}{l}\text { réclames, } \\
\text { textes pro- } \\
\text { motionnels }\end{array}$ & \\
\hline TEXTOLOGIE & textes littéraires & & & \\
\hline ANALYSE de CONTENU & articles de presse & & $\begin{array}{l}\text { brochures } \\
\text { touristiques }\end{array}$ & \\
\hline $\begin{array}{c}\text { ANALYSE } \\
\text { CONVERSATIONNELLE }\end{array}$ & $\begin{array}{c}\text { interactions/textes } \\
\text { spécialisés }\end{array}$ & & interviews & \\
\hline
\end{tabular}

Habituellement, on détaille ces stratégies ou techniques de traduction (condensation, explicitation, transcodage, expansion, reformulation, adaptation ponctuelle, omission, transmutation, paraphrase, compensation, emprunt, traduction littérale, 
etc.). Par contre, rien ou presque n'est suggéré comme stratégies de lecture. Très souvent, les apprenants traducteurs se bloquent sur un mot inconnu, ou prennent prétexte d'un mot inconnu pour dire leur incompréhension - oubliant ainsi qu'un texte fait sens non seulement avec des facteurs extra-linguistiques mais aussi avec des facteurs intertextuels (présuppositions, isotopie sémantique, structure des paragraphes, traits suprasegmentaux, marques de cohésion, etc.).

Les diverses analyses de discours (cf. van Dijk, 1985; Maingueneau, 1995) sont aujourd'hui un ensemble hétérogène quant aux traditions et filiations revendiquées, quant aux présupposés théoriques, quant aux corpus et aux méthodes, quant aux visées, quant à l'organisation sociale même des chercheurs. Cette diversité ainsi que l'ouverture vers la sociologie, la psychologie, l'histoire, les théories de l'argumentation, la logique... peuvent rebuter le jeune traducteur-lecteur; elles peuvent également le décomplexer, dans un relativisme de bon aloi.

Dans le tableau ci-dessous, on croise des modèles ou approches d'AD avec des exemples de genres de texte à traduire - regroupés sous trois hyper-catégories. Cette convergence entre une partie des sciences du langage - qui devraient diversifier au plus vite leurs corpus pour tenir compte de la pluralité des supports actuels - et la pédagogie de la traduction facilite à n'en pas douter la maîtrise d'une des compétences fondamentales du traducteur: la capacité d'analyse de messages de plus en plus multisémiotiques (verbal, iconique, sonore, graphique, etc.) - messages rendus sous des noms différents (traduction, adaptation, rédaction multilingue, editing, localisation, etc.) parce que le marché tend à assimiler «traduction» à «version mot à mot»! La convergence pose avec acuité le problème de la formation des formateurs, habitués trop souvent à la division académique des disciplines.

\section{EN GUISE DE CONCLUSION}

Des travaux qui essaient de combiner réflexion traductologique et analyses de discours, on peut percevoir de nombreux non-dits. Par exemple Delisle (1980) ne s'attarde pas sur ce qu'il entend ou sur ce qu'il faut entendre par «discours»; Hatim et Mason (1990) de même mais pour «traduction». Dans une perspective d'enseignement, la question n'est peut-être pas de première importance. Ici même, on a amalgamé discours et texte, alors que bien des distinctions opératoires existent - rattachant le «discours» par exemple à un processus, à de l'oral, à des procédures de négotiation, à une dynamique communicationnelle tandis «texte» serait relié au produit statique, clos, à de l'écrit, à une organisation séquentielle, etc.

Par ailleurs, on a limité le «discours» à la source du travail du traducteur, sans nous interroger sur la valeur heuristique du concept par rapport au texte d'arrivée. S'agit-il de deux «discours»? La traduction a certainement son mot à dire comme procédure de découverte sur l'analyse du discours interculturel. Empruntant aux sciences du langage, elle aurait avec son regard décalé un rôle actif à jouer pour les 
féconder, notamment quand il nous faut envisager les communications internationales dans un univers de plus en plus global, quand il nous faut construire une politique linguistique pour une société multilingue/multiculturel. Ce serait une vraie interdisciplinarité.

\section{BIBLIOGRAPHIE}

A rntz, R. (éd) (1988), Textlinguistik und Fachsprache, Hildesheim: Olms.

B aker, M. (1992), In other words, London-New York: Routledge.

B as tu ji, J. (1974), Traduction et théorie linguistique, Change, numéro 19, p. 25-45.

B ell, R. (1991), Translation and Translating: Theory and Practice, London-New York: Longman.

Catford, J. C. (1965), A Linguistic Theory of Translation, London: Oxford U.P.

Chesterman, A. (1997), Memes of translation, Amsterdam: J. Benjamins.

Dancette, J. (1995), Parcours de traduction: étude expérimentale du processus de compréhension, Lille: P.U. de Lille.

Deli sle, J. (1980), L'analyse du discours comme méthode de traduction, Ottawa: University of Ottawa Press.

Folkart, B. (1991), Le conflit des énonciations: traduction et discours rapporté, Québec: Balzac.

Foucault, M. (1971), L'ordre du discours, Paris: Gallimard.

Garnie r, G. (1985), Linguistique et traduction, Caen: Paradigme.

Guillemin-Flescher, J. (1981), Syntaxe comparée du français et de l'anglais: problèmes de traduction, Paris: Ophrys.

Gutt, E.-A. (1991), Translation and Relevance: Cognition and Context, Oxford: Basil Blackwell.

Hartman n, R. (1980), Contrastive Textology: Comparative Discourse Analysis in Applied Linguistics, Heidelberg: Julius Groos.

Hatim, B. et Mas on I. (1990), Discourse and the Translator, London-New York: Longman.

Hatim, B. et Mas on I. (1997), Communication across cultures: Translation and Contrastive Text Linguistics, Exeter: Exeter U.P.

House, J. (1981), A Model for Translation Quality Assessment, Tübingen: Gunter Narr.

Hous e, J. et B l u m-Kulka, S. (eds) (1986), Interlingual and Intercultural Communication: Discourse and Cognition in Translation and Second Language Acquisition Studies, Tübingen: Gunter Narr.

Hurtado Albir, A. (1996), La traduction: classification et éléments d'analyse, Meta 41 (3), 366-377.

Jakobson, R. 1959 (1971), On Linguistic Aspects of Translation in: Brower, R. A. (ed.): On translation, Cambridge, MA: Harvard U.P. p. 232-239.

Koptjevskaja-Tamm, M. (1989), Linguistic translation theory in Soviet Union(1950-1980's), Stockholm: TÖI - Université de Stockholm.

Küh I we in et al. (1981), Kontrastive Linguistik und Übersetzungswissenschaft. Munich: Wilheim Fink verlag.

Ma inguen eau, D. (éd) (1995), Les analyses du discours en France, Langages, numéro 117.

Moun in, G. (1976), Linguistique et traduction, Bruxelles: Dessart et Mardaga.

Neubert, A. (1985), Text and Translation, Leipzig: VEB Verlag Enzyklopädie.

New mark, P. (1981), Approaches to Translation, Oxford: Pergamon Press. 
Nida, E. (1964), Towards a Science of Translating: With Special Reference to Principles and Procedures Involved in Bible Translating, Leiden: E.J. Brill.

Nid a, E. et Taber, C. (1969), The Theory and Practice of Translation, Leiden: E.J. Brill. Nord, C. (1991), Text Analysis in Translation, Amsterdam, Atlanta, GA: Rodopi.

Pếch eux, M. (1975), Les vérités de la Palice: linguistique, sémantique, philosophie, Paris: Maspéro.

Reiss, K. (1976), Texttyp und Übersetzungsmethode. Der Operative Text, Kronberg: Scriptor. Rolf, E. (1993), Die Funktionen der Gebrauchstexsorten.

Snell-Horn by, M. (1988), Translation Studies: an Integrated Approach, Amsterdam: J. Benjamins.

Švejcer, A. (1986), Übersetzung und Linguistik (trad. du russe), Berlin: Akademie-Verlag.

Tabakowska, E. (1993), Cognitive Linguistics and Poetics of Translation, Tübingen: Gunter Narr.

Tirkkonen-Condit, S. (1986), Text Type Markers and Translation Equivalence, House et Blum-Kulka (eds), p. 95-113.

Toury, G. (1995), Descriptive Translation Studies and Beyond, Amsterdam: J. Benjamins.

Trosborg, A. (ed.) (1997), Text Typology and Translation, Amsterdam: J. Benjamins.

Van Dijk, T. (ed.) (1985), Handbook of Discourse Analysis (4 volumes), London: Academic Press.

V an Dijk, T. et W. Kintsch (1983), Strategies of Discourse comprehension, London: Academic Press.

Vin ay, J. P. et Darbelne t, J. (1958), Stylistique comparée du français et de l'anglais: méthode de traduction, Paris: Didier. 